\title{
Energy Efficient Window Control Scheme for IEEE 802.11ah (Wi-Fi HaLow) Based Networks
}

\author{
Yanru Wang, Kok Keong Chai, Yue Chen and John Schormans \\ School of Electronic Engineering and Computer Science, Queen Mary University of London, London, UK
}

\begin{abstract}
IEEE 802.11ah brings in Restricted Access Window (RAW) to decrease contention, which is the grouping-based MAC protocol. The way to group a large number of devices and application of RAW size would have influence on the energy efficiency in the process of medium access and communications. In this paper, we study an efficient window control algorithm to improve the uplink energy efficiency with a novel retransmission scheme that utilises the next empty slot for retransmission in the uplink. The grouping scheme is based on the college admission game. The problem is formulated based on energy efficiency by probability theory and Markov chain. To optimise energy efficiency, a window control scheme is proposed to group the devices and set the adaptive window size (number of slots per RAW and internal slot interval) based on the number of groups, applications and the distance between devices and Access Point (AP). The optimal solution is derived by Gradient Descent approach. Simulation results show that our proposed algorithm outperforms existing one on uplink energy efficiency and fairness.
\end{abstract}

Key words: IEEE 802.11ah, MAC, RAW, uplink energy efficiency, grouping, adaptive window.

\section{Introduction}

One of the latest wireless communication technologies that have been proposed for Internet of Things (IoT) applications is the Low Power Wi-Fi [1]. IEEE 802.11 ah WLAN standard group has put forward IEEE 802.11ah to support Low Power Wi-Fi (called "Wi-Fi HaLow"), which stated at 2011 [2, 3]. IEEE 802.11ah operates at sub-1 GHz and it can support up to 6,000 devices within a network with transmission range up to $1 \mathrm{~km}$ at the rate of more than $100 \mathrm{kbps}$ [1]. Restricted Access Window (RAW) is a new MAC layer feature that used in Low Power Wi-Fi to decrease collision. It limits a set of devices to access the channel at any time and spreads their attempts over a period of time [2]. RAW consists of multiple equal time slots, where each slot is selected by devices or assigned to a group of devices $[1,4]$. Devices are in wake-up mode only when turning to their RAW, otherwise would be in doze mode.

Corresponding author: Yanru Wang, Ph.D. candidate, research fields: energy efficient channel access control, IEEE 802.11ah, Internet of Things (IoT), energy efficiency in IoT networks.
The window control strategy has the effect on the energy efficiency of the whole network communications. The number of devices in the group influences the collision probability. The energy consumption is related to the distance of each device. And there is no standard definition for RAW duration. For one RAW with long duration, the devices involved should be in active mode for a longer period, leading to expending idle wake-up energy. On the contrary, the collision probability would be high if a large number of devices access through RAW with limited time slots, resulting in low efficiency. Adaptive internal time slot duration can ensure sufficient time to access and transmit, but also avoid needless idle time. And RAW has an effect on overhead information to inform scheduling information. Meanwhile, since the device selects time slots randomly in uplink communication, there are some empty slots that no device to choose, while some slots are selected by multiple devices, which will result in collision. Retransmission mechanism for collided devices is a way to reuse the empty slots. So an energy efficient window control scheme for grouping dense devices and setting 
adaptive window size is required in IEEE 802.11ah based IoT networks to optimise energy efficiency.

The way to improve energy efficiency has been studied in depth in many research works, mainly focus on improving successful transmission probability and reducing collision [12], a load-balanced sensor grouping algorithm is proposed to group the devices to improve channel utilisation. In Ref. [4], a new medium access control enhancement algorithm was proposed to determine optimal size of RAW by applying Maximum Likelihood (ML) estimation method to estimate the number devices for uplink access. In Ref. [5], the authors introduced Successive Interference Cancellation to improve the throughput in limited time but resulting in more collisions. In Refs. [6, 7], new algorithms were proposed to estimate wake-up time of devices. In Ref. [8], less collision was achieved by limiting the number of devices in authentication stage. In Ref. [9], the RAW mechanism is analysed based on non-cross slot boundary holding scheme. Our previous work [10] proposed adaptive RAW for IEEE 802.11 ah based networks. The aim is to achieve highest uplink energy efficiency by estimating optimal combination of the number of time slots and proper group size involved for one uplink RAW. Out latest work [13] represented novel retransmission scheme and an algorithm for AP to set proper window size to jointly optimise energy efficiency and delay. However, we have not considered the grouping strategy and distance between devices and AP.

The main contribution of these papers is to improve energy efficiency based on transmission probability and collision for IEEE 802.11ah. Less work has been done to optimise both grouping method and adaptive window to improve the energy efficiency. To address the above consideration, in this paper we study an optimisation problem aiming at maximising uplink energy efficiency through grouping and setting adaptive window for dense devices. An energy efficient window control algorithm is proposed to determine the grouping strategy and window size for the groups in one scenario based on total number of groups and application types. An optimal solution is derived by applying college admissions games and Gradient Descent approach.

The remainder of the paper is organised as follow. Section 2 describes system model about IEEE 802.11 ah MAC protocol. A college admissions game for clustering is given in Section 3. Section 4 presents the energy efficiency formulation. Section 5 shows the proposed window control scheme for grouping strategy and window setting. Section 6 provides the simulation results to show the efficacy of the proposed scheme. Finally, Section 7 concludes the paper.

\section{System Model}

We consider a single-hop topology for dense IEEE802.11ah IoT networks as a single Access Point (AP) with a high number of devices. RAW groups the devices and splits the channel into equal time slots. All devices listen in the beginning of beacon frame called Target Beacon Transmission Time (TBTT) to obtain scheduling information that indicates which RAW they belong to. Then devices fall into sleep mode until turning to their RAW to attempt accessing. For gth RAW, there are $M$ time slots and $N$ devices limited by lowest and highest associated identifier (AID) of devices which indicates the location, traffic, type, energy saving mode etc. [1].

In IEEE 802.11ah uplink communications, the devices who have buffered uplink data randomly select a time slot in allocated RAW and attempt to access the channel as shown in Fig. 1. In the selected slot, it applies CSMA with Collision Avoidance (CSMA/CA), which is a sort of listen before talk mechanism. The devices sense the channel to determine whether the channel is busy before initiating a transmission. If the medium is idle during a period time which called the Distributed Inter-Frame Space (DIFS), the device is allowed to transmit. If the medium is sensed busy, the transmission is delayed until the channel is idle again. A slotted binary exponential back-off interval is 


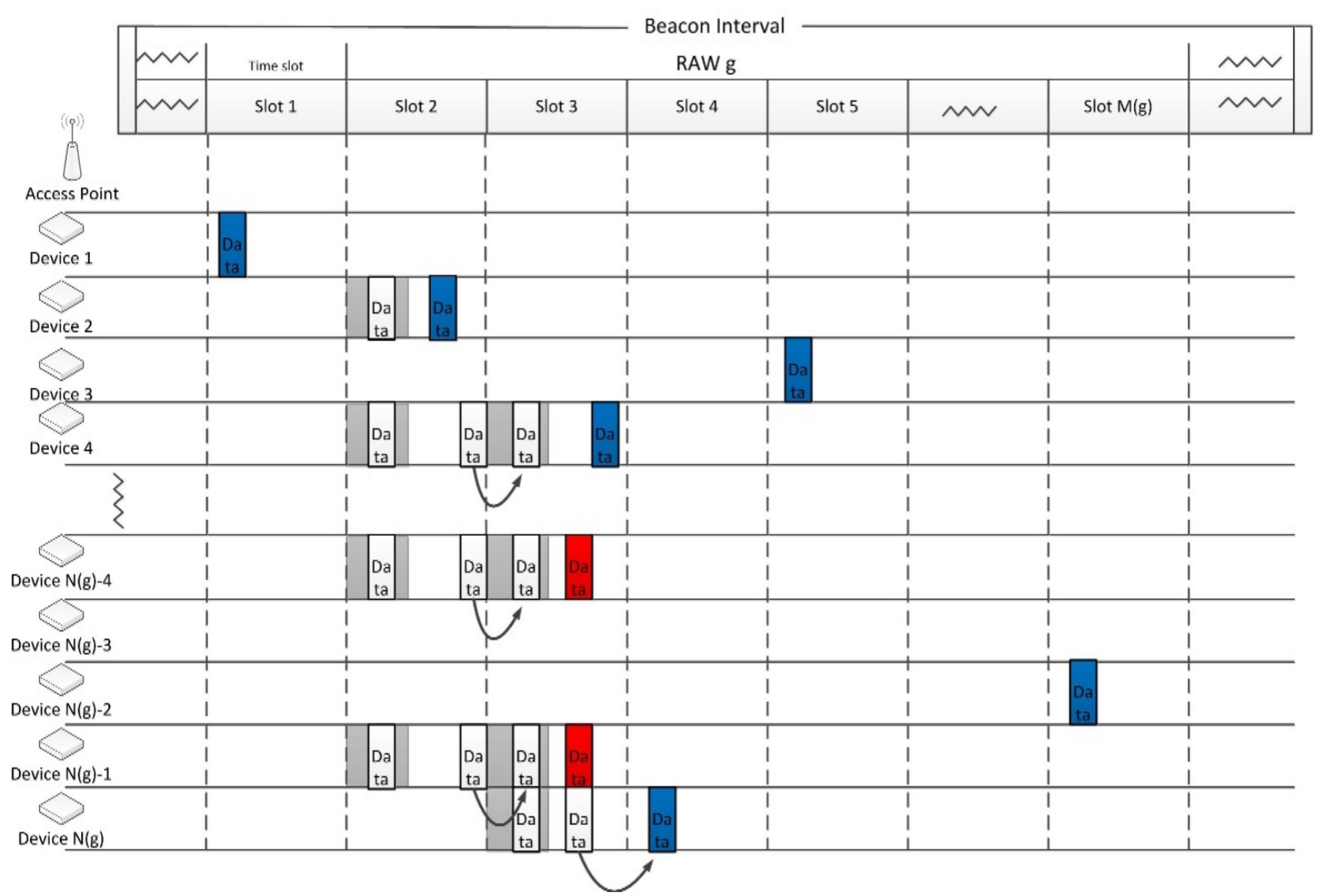

Fig. 1 Operation of RAW.

uniformly chosen in contention window. So after random selection, if there is only one device in a slot, it could access directly as Device 1 transmits packet in Slot 1 directly without contention, and the same for Device 3 in Slot 5 and Device $\mathrm{N}(\mathrm{g})-2$ in Slot $\mathrm{M}(\mathrm{g})$. When there are more than one device choosing the same time slot, for example, Device 2, Device 4, Device N(g)-4, Device N(g)-1 in Slot 2, they would come into the back-off stage to avoid collision by doubling contention window and trying again until reaching the slot boundary. If accessing the channel successfully, device requests uplink communication by sending Power Save-poll message to the AP. AP responses with an ACK to confirm connection. After the first handshake, the device transmits buffered data frame and waits for ACK from AP [11]. We assume if not accessing the channel, it would retransmit in the next slot, for example, when Device 2 has accessed the channel in Slot 2, Device 4, Device N(g)-4 and Device
$\mathrm{N}(\mathrm{g})-1$ retransmit in Slot 3, which needs to contend with Device $\mathrm{N}(\mathrm{g})$. But when they cannot retransmit successfully in the following slot, they need to retransmit in subsequent allocated RAW, such as Device N(g)-4 and Device N(g)-1 in Slots 3 and 4. The process is repeated, one RAW by one RAW, until the end of beacon frame.

\section{College Admission Game for Clustering in IEEE 802.11ah}

The college admission games, also known as many-to-one matching games, are applied to clustering the devices for the super dense networks. The many-to-one matching models the students to apply for several colleges, and the each college involves a certain quota for the number of students that it can admin. The advantages of matching theory include suitable models for characterising the interactions between heterogeneous nodes, the ability to define 
general preferences, suitable solution in terms of stability and optimality and efficient algorithm implementations.

This framework is suitable for the proposed problem as the different distances between devices and AP while characterising stable and desirable clustering devices. We formulate college admissions game through three components: (1) the set of devices acting students; (2) the set of groups with quota $q$ for each group acting school; (3) the preference list of devices is built based on contention factor while that of groups is built based on the average distance.

\section{Adaptive Window for IEEE 802.11ah}

In this section, we formulate energy efficiency for IEEE 802.11ah uplink communications. The process of devices to access channel in IEEE 802.11ah is formulated by Markov Chain and the energy efficiency is derived.

For the uplink communication in MAC layer of IEEE $802.11 \mathrm{ah}$, the number of devices to contend is limited by RAW. Time slots in RAW are selected by devices randomly. Since random selection, the states of each time slot are different, and retransmission makes it depend on the state of previous slot, so Markov Chain is applied to monitor accessing process of each time slot in one RAW.

The process in Markov Chain is represented by three parameters $j, \mathrm{n}_{j}, s_{j}$, where $j$ is the index of time slot in one RAW; $\mathrm{n}_{j}$ shows there are $\mathrm{n}_{j}$ devices to choose the $j$ th time slot; $S_{j}$ is the number of devices accessing channel successfully, which is illustrated in Fig. 2. If number of devices in one slot is the combination of the devices that choose this slot in random selection and

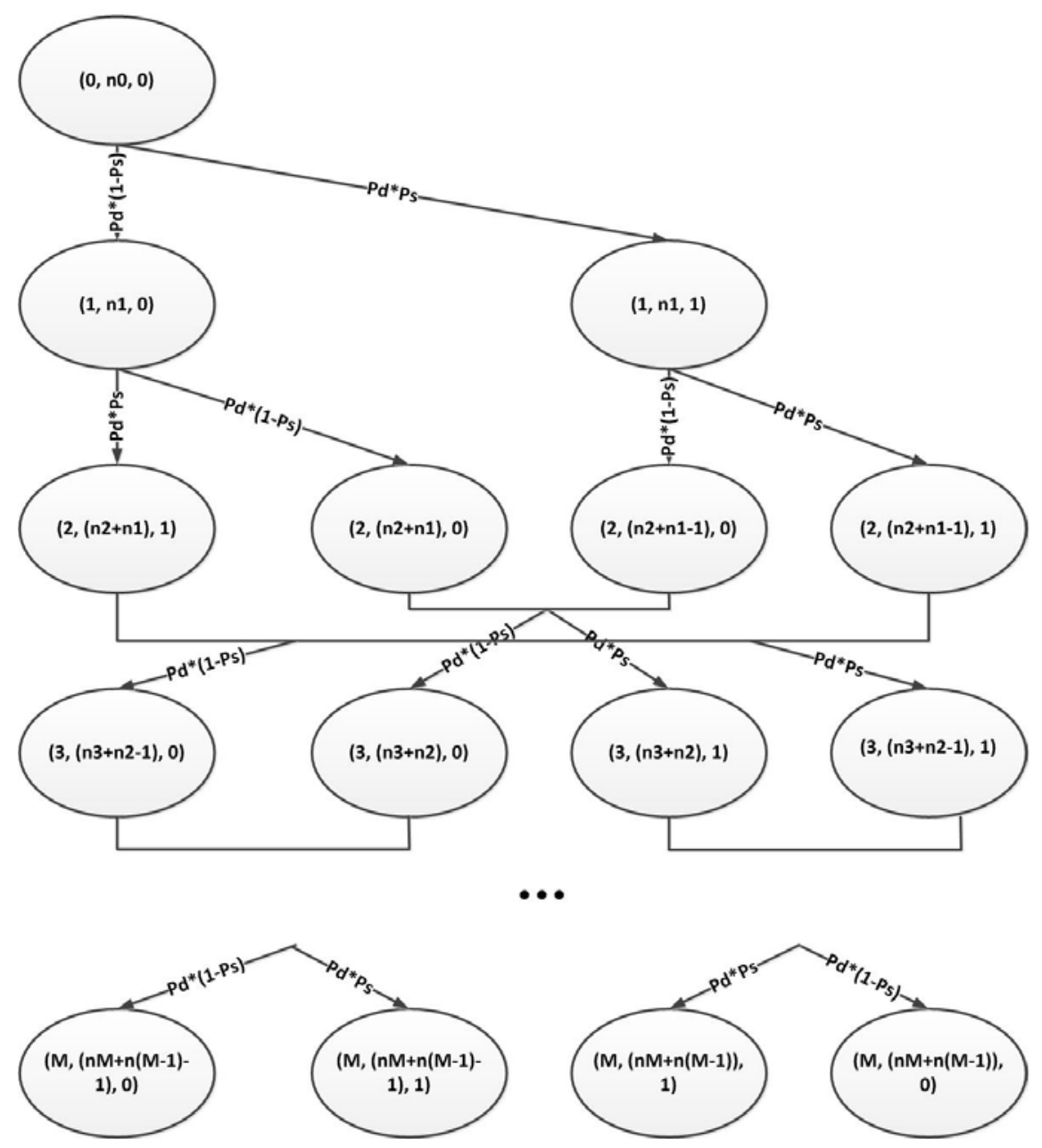

Fig. 2 Markov chain. 


$$
\begin{aligned}
& P_{i j}= \begin{cases}\mathrm{P}_{d_{-} n_{j}} \mathrm{P}_{\mathrm{s}_{-} n_{j}}, & \text { if }\left(\mathrm{i}=0,0<n_{j}<\mathrm{N}, \mathrm{s}_{j}=1\right) \\
\mathrm{P}_{d_{-} n_{j}}\left(1-\mathrm{P}_{\mathrm{s}_{-} n_{j}}\right), & \text { if }\left(\mathrm{i}=0,0<n_{j}<\mathrm{N}, \mathrm{s}_{j}=0\right) \\
\mathrm{P}_{d_{-} n_{j}} \mathrm{P}_{\mathrm{s}_{-}\left(n_{j}+n_{i}-s_{i}\right)}, & \text { if }\left(\mathrm{i}>0,0<n_{j}<\left(\mathrm{N}-\sum_{k=0}^{i-1} s_{k}\right), \mathrm{s}_{j}=1\right) \\
\mathrm{P}_{d_{-} n_{j}}\left(1-\mathrm{P}_{\mathrm{s}_{-}\left(n_{j}+n_{i}-s_{i}\right)}\right), & \text { if }\left(\mathrm{i}>0,0<n_{j}<\left(\mathrm{N}-\sum_{k=0}^{i-1} s_{k}\right), \mathrm{s}_{j}=0\right) \\
0, & \text { otherwise }\end{cases} \\
& P_{s_{-} n_{k} \_ \text {backoff }}=\sum_{k=0}^{W_{\min }-1}\left\{\prod_{0}^{k}\left[1-\frac{1}{W_{\min }}\left(1-\frac{k}{W_{\min }}\right)^{n_{k}-1}\right]\right\} \frac{1}{W_{\min }}\left(1-\frac{k+1}{W_{\min }}\right)^{n_{k}-1},
\end{aligned}
$$

the collided devices from previous one slot, so for $j$ th slot, the contending devices is $n_{j}+n_{j-1}$. If $(j-1)$ th slot has no packet transmitted, otherwise the contending devices are $n_{j}+n_{j-1}-1$.

The transition probability of adjacent states could be denoted as Eq. (1), where $P_{i j}$ is the probability to transfer from slot $i$ to slot $j ; \mathrm{P}_{d_{-} n_{j}}$ is the probability of there are $n_{j}$ devices to choose the same time slot; $\mathrm{P}_{\mathrm{s}_{-} n_{j}}$ the probability of successfully accessing.

As for a time slot in RAW, the probability that there are $n_{k}$ devices to choose is

$$
P_{d_{-} n_{k}}=\left(\begin{array}{l}
N \\
n_{k}
\end{array}\right)\left(\frac{1}{M}\right)^{n_{k}}\left(1-\frac{1}{M}\right)^{N-n_{k}},
$$

where $M$ is the number of uplink time slots contained in one RAW; $N$ is the number of devices that could be involved in one RAW intending to access channel; $n_{k}$ is the number of devices to choose the $k$ th time slot.

According to Ref. [4], there are two cases for a device to transmit uplink packet successfully in one slot.

Case 1: a time slot chosen by only one device. This device would transmit a packet successfully without other contending devices.

Case 2: a time slot chosen by multiple devices. The devices will go into back-off stage due to contention, and one of them will succeed in accessing the channel at the first back-off stage.
So the successful accessing probability could be described as

$$
P_{s_{-} n_{k}}=\left\{\begin{array}{l}
1, \text { if }\left(n_{k}=1\right) \\
P_{s_{-} n_{k} \text { backoff }}, \text { if }\left(\mathrm{n}_{k}>1\right)
\end{array}\right.
$$

where $n_{k}=1$ denotes the situation as Case $1 ; \mathrm{n}_{k}>1$ is related to Case 2; $P_{s_{-} n_{k_{-}} \text {backoff }}$ is the probability of access channel in first back-off stage in Case 2.

For Case 2, in view of $(i>1)$ other contending devices, only one device will success in accessing the channel at the first back-off. The probability of a minimum contention window as the first back-off stage is Eq. (4), where $W_{\min }$ is the minimal size of contention window.

The total number of packets that could be sent in one RAW is

$$
\sum_{0}^{M} s_{k}=\sum_{0}^{M} s_{k_{-} 1}+\sum_{0}^{M} s_{k_{-} 2}
$$

where $\sum_{0}^{M} s_{k_{-} 1}$ is the number of packets sent as Case 1; $\sum_{0}^{M} s_{k_{-}}$is the number of packets sent as Case 2. is

The transmission energy consumption of one RAW

$$
\begin{aligned}
& E_{t}=E_{t 1} \sum_{0}^{M} s_{k_{-} 1} l_{s}+E_{t 2} \sum_{0}^{M} s_{k_{-} 2} l_{s}+ \\
& E_{t 3}\left(N l_{s}-\sum_{0}^{M} s_{k} l_{s}\right)+E_{c o n} M \tau N,
\end{aligned}
$$


where $E_{t 1}$ is the energy consumption rate when transmitting a packet as Case 1 for $1 \mathrm{~km} ; E_{t 2}$ is the energy consumption rate when transmitting a packet as Case 2 for $1 \mathrm{~km} ; E_{t 3}$ is the energy waste rate when there is collision both in its slot and next slot so that it needs to retransmit in another RAW for $1 \mathrm{~km} ; E_{\text {con }}$ is the contention power in one RAW, which is the energy consumed when it is in wakeup mode; $\tau$ is the time duration of one time slot; and $l_{s}$ is the distance between a certain device and AP.

The size of RAW also determines the energy consumption of transmitting overhead information, because when the window duration is too small, the overhead information of each device would be high due to the scheduling information that needs to be transmitted multiple times in a short time. And if the number of devices involved in one RAW is small, it also needs massive scheduling information to realise network communication. So the energy consumption of overhead information is related to $N$ and $M$ :

$$
E_{\text {head }}=\frac{\alpha}{M} \times \frac{\beta}{N},
$$

where $\alpha$ is the parameter indicating traffic and $\beta$ is the parameter related to overall number of devices in the scenario.

Energy efficiency of one RAW could be evaluated by the data rate it provides and overall energy consumption. Data rate could be formulated as

$$
\begin{aligned}
& s_{1}=\sum_{n_{1}=1}^{N} P_{d_{-} n_{1}} P_{s_{-} n_{1}}
\end{aligned}
$$

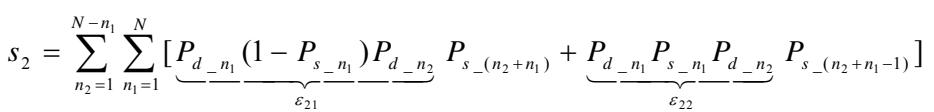

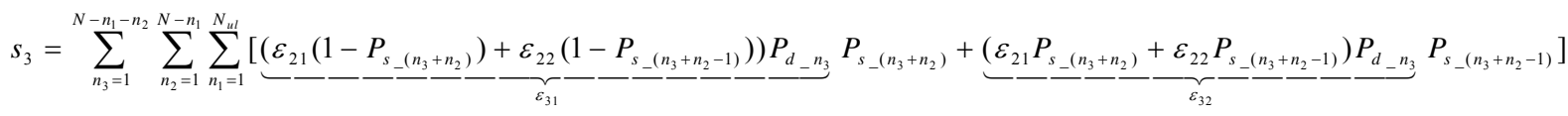

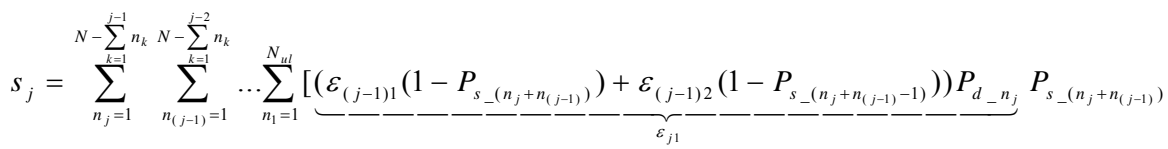

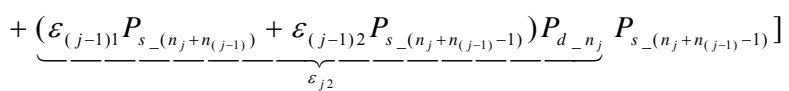

In order to estimate number of packets transmitted successfully, the average numbers of packets that could be transmitted successfully are formulated as where $\gamma$ is the packet size. $\gamma \sum_{0}^{M} s_{k}$ is the total length of packets could be transmitted for $N$ devices in $M$ time slots. $\tau M$ is the total time of one RAW.

The overall energy consumption consists of transition power and overhead power when $N$ devices attempt to communicate with AP during one RAW, which could be denoted by

$$
E_{\text {overall }}=E_{t}+E_{\text {head }} \text {. }
$$

Thus energy efficiency is

$$
E E=\frac{R}{E_{\text {overall }}}=\frac{\gamma \sum_{0}^{M} s_{k}}{\tau M\left(E_{t}+N E_{\text {head }}\right)} .
$$

The average delay (waiting time from the beginning the packets transmitted in one RAW is

$$
D=\sum_{0}^{M} k \tau s_{k}\left(\sum_{0}^{M} s_{k}\right)^{-1} .
$$

So the energy efficiency could be denoted as

$$
E E=\frac{R}{E_{\text {overall }}}=\frac{\gamma \sum_{0}^{M} k \tau s_{k}}{\tau M D\left(E_{t}+N E_{\text {head }}\right)} .
$$
of RAW to the slot that the device can access) for all

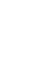


The optimisation problem could be formulated as

$$
\begin{aligned}
& \max _{g=1}^{G_{t}} \mathrm{EE}_{u l_{-} g}=\frac{\gamma \sum_{0}^{M} k \tau s_{k}}{\tau M D\left(E_{t}+\left|\mu\left(G_{g}\right)\right| E_{\text {head }}\right)} \\
& \text { s.t } \\
& \sum_{\mathrm{g}=1}^{G_{t}} \sum_{j=1}^{q} G_{k j} \geq N_{t} \\
& \mid \mu\left(\text { Device }_{i}\right) \mid=1 \\
& \left|\mu\left(G_{g}\right)\right|<q
\end{aligned}
$$

where $\mu$ means the matching as $\mid \mu\left(\right.$ Device $\left._{i}\right) \mid=1$ is one device can match to one group and $\left|\mu\left(G_{g}\right)\right|<q$ means the devices in one group can not extend the quota.

Thus energy efficiency is a function related to the number of devices involved $(N)$ and time slot in one RAW $(M, \tau)$. We could maximise energy efficiency by finding optimal $M$ and $\tau$ based on diverse groups.

\section{Energy Efficient RAW Control}

We find the energy efficient window control scheme by college admission game and Gradient Descent approach, as shown in Algorithm 1.

Algorithm 1 Gradient Descent Window Control Scheme with College Admission Game for Super Dense Networks

Input

Set of devices Device

Number of devices Device $_{t}$

Set of groups $G$

Total number of groups $G t$

Quota for each group $q$

\section{Sept 1: Initial network.}

The network starts with an initial assignment at the beginning of all time no user is assigned to any groups.

Random assign $n$ devices to each group.

Remove assigned $n G t$ from set of devices.

Step 2: College admission game for devices clustering.

while $G \neq \varnothing$ do

Select $n^{\prime}$ devices from Device.

$n$ ' devices and $G t$ groups build their own preference list individually.

loop

$n$ ' devices send the application to groups based on preference list.

$G t$ groups accept or reject the application based on their preference list.

If Device $_{i}$ accepted by $\mathrm{G}_{j}$, it denotes as $\mu\left(\mathrm{G}_{j}\right)=$ Device $_{i}$.

And Device $\leftarrow$ Device $\backslash\left\{\right.$ Device $\left._{i}\right\}$, and all groups remove Device $_{i}$ from its preference list.

If $\left|\mu\left(\mathrm{G}_{j}\right)\right|=q$, all devices remove $\mathrm{G}_{j}$ from its preference list.

end loop

end while

Step 3: The uplink window size is determined according to the number of uplink devices in one group. $N_{g}=\left|\mu\left(\mathrm{G}_{g}\right)\right|$

Step 3.1: The uplink time slot durations are set based on the type of devices, which are the constraints. uplink time slot duration $\geq$ transmit time + back off time; 


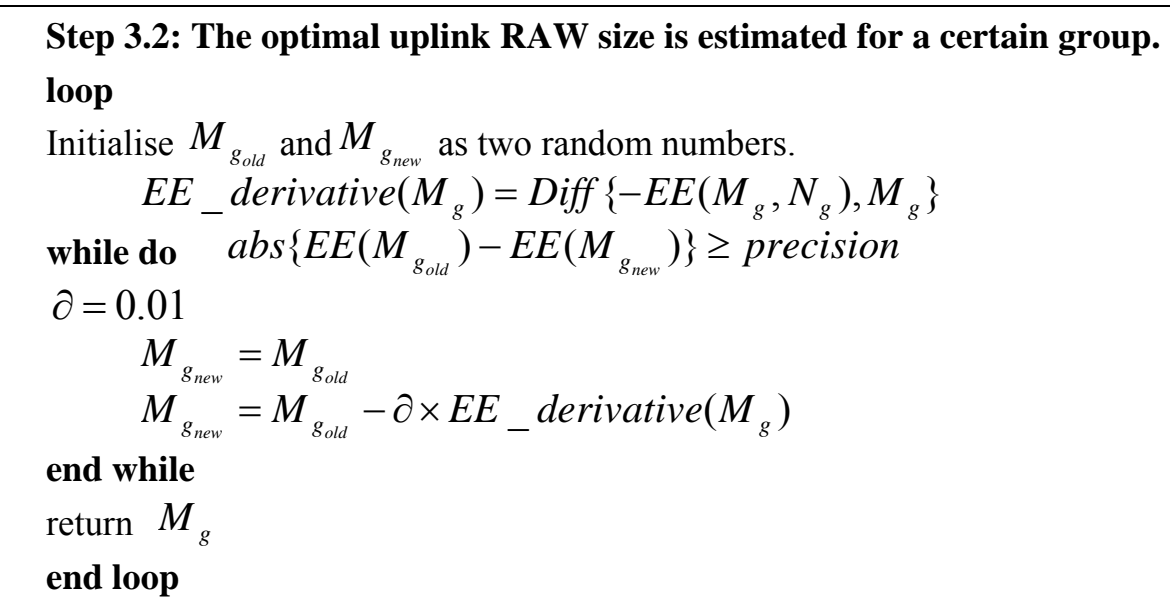

Step 3.3: Set RAW as $M_{g}$ time slots for this group. The devices fall into sleep mode and wake up until coming to their allocated window.

Step 3.4: $N_{g}$ devices randomly select one of $M_{g}$ timeslots in RAW and attempt to access channel.

When the device could not access in its slot, re-attempt in the following slot. If it cannot access in next slot, reattempt in the next allocated window.

Table 1 Simulation parameters.

\begin{tabular}{llll}
\hline Parameter & Value & Parameter & Value \\
\hline Frequency & $0.9 \mathrm{GHz}$ & Data rate & $100 \mathrm{kbps}$ \\
Maximal distance & $1 \mathrm{~km}$ & Total number of devices & 6,000 \\
$E_{t 1}$ & $2.692 \mathrm{mw} / \mathrm{km}$ & $E_{t 2}$ & $5 \mathrm{mw} / \mathrm{km}$ \\
$E_{t 3}$ & $6 \mathrm{mw} / \mathrm{km}$ & Idle listen power & $0.001 \mathrm{mw}$ \\
Min contention window & 8 & Max contention window & 1,024 \\
$\alpha$ & 200 & $\beta$ & 200 \\
\hline
\end{tabular}

This algorithm is divided into three main parts to find the optimal window solution. At initialisation stage, it is to randomly put some devices into each group. The second step is to cluster devices by applying college admissions game. The third step is to set the adaptive window size based on group by Gradient Descent approach. It starts with an arbitrary value based on a random value, and then compares the existing optimum with new function results with input adding a gradient value. When upcoming one performs better, accept new one, otherwise, reserve previous. Step by step to find the optimal value according to gradient descent route.

\section{Simulation Results and Analysis}

In this section, the energy efficient window control scheme is evaluated in Matlab. We consider a one-hop topology as described in the system model and apply the proposed window control algorithm to group dense devices and determine window sizes for different groups in the scenario. The nodes in the scenario are following normal distribution which means higher proportion of devices is close to AP and less devices locate at the boundary of scenario.

We apply wake-up/sleep mode for all devices. When coming to allocated RAW, devices keep wake-up mode and randomly select their own time slot to do transmission to the AP, otherwise they would fall into sleep mode. We assume that every device involved has exactly one packet to transmit for uplink communications during one RAW. The performances are evaluated based on the total number of groups per scenario as 6,000 devices are divided into how many groups. The main simulation parameters are given in Table 1. 
The standard deviation comparison of number of devices pre group for diverse number of groups per scenario is shown in Fig. 3. It can be observed that the standard deviation of proposed grouping scheme is lower than random grouping, which means the number of devices pre group is more similar for one scenario, so the proposed one improves the fairness for clustering than random grouping. The standard deviation of proposed one is nearly 0 when the number of groups is divisible by 6,000 (the total number of devices in one scenario) such as 50, 60, 75 and 80, as group sizes are nearly same under this situation. The curve of random grouping declines with increasing the number of groups, since there are more choices for devices to choose so that the numbers of devices pre group would not be large gap, while for less number of groups, the probability for big difference of the sizes between two groups is high.

The uplink energy efficiency is evaluated based on the number of groups per scenario and the application types (packet sizes).

The uplink energy efficiency per RAW varying over diverse number of groups with 512 bits/packet is shown in Fig. 4. The proposed window control is to group the devices efficiently and set the best matched window size and internal slot interval based on group to achieve optimal energy efficiency with the novel retransmission scheme as the collided devices retransmit in next slot. The existing grouping method is random grouping, and the existing RAW is to set the adaptive number of slots based on optimisation of energy efficiency and the retransmission is in another allocated RAW when there is a collision [10]. It can be observed that the proposed RAW performs better than the existing one with $2.0 \%$ improvement for existing grouping with adaptive window and $141 \%$ improvement for proposed grouping scheme with existing window.

For proposed grouping scheme, the fairness for devices improves so that the overall contention is lower than the random grouping, which is the reason to result in higher energy efficiency. As random access in uplink communication, there are many empty slots without device to choose. The proposed RAW with retransmission scheme is to retransmit in next lot for collided devices in crowded slot (selected by more than one device), which could reuse the empty slots, so there are more packets that could be transmitted in proposed window control, leading to high energy efficiency. The adaptive time slots could decrease the wake-up power, which is a way to reduce the energy consumption, thus the proposed window control could outperform than the existing RAW. When the number of groups is low,

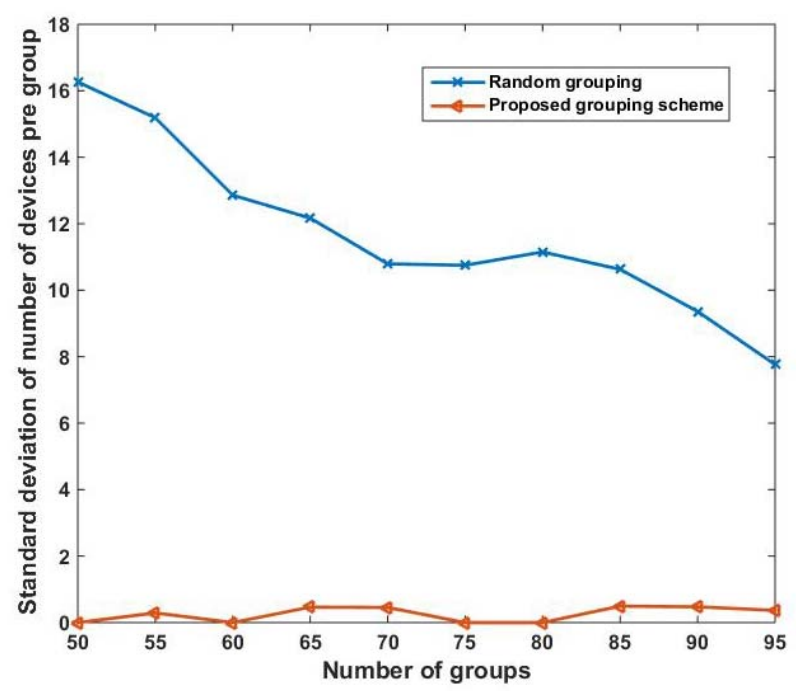

Fig. 3 Standard deviation of number of devices pre group.

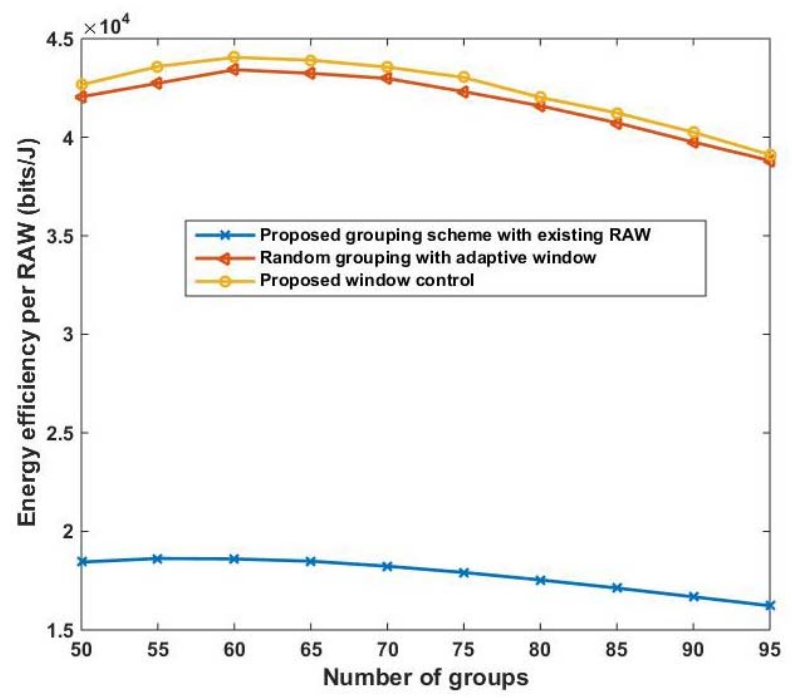

Fig. 4 Uplink energy efficiency per RAW comparison with various number of groups in one scenario (512 bits/packet). 


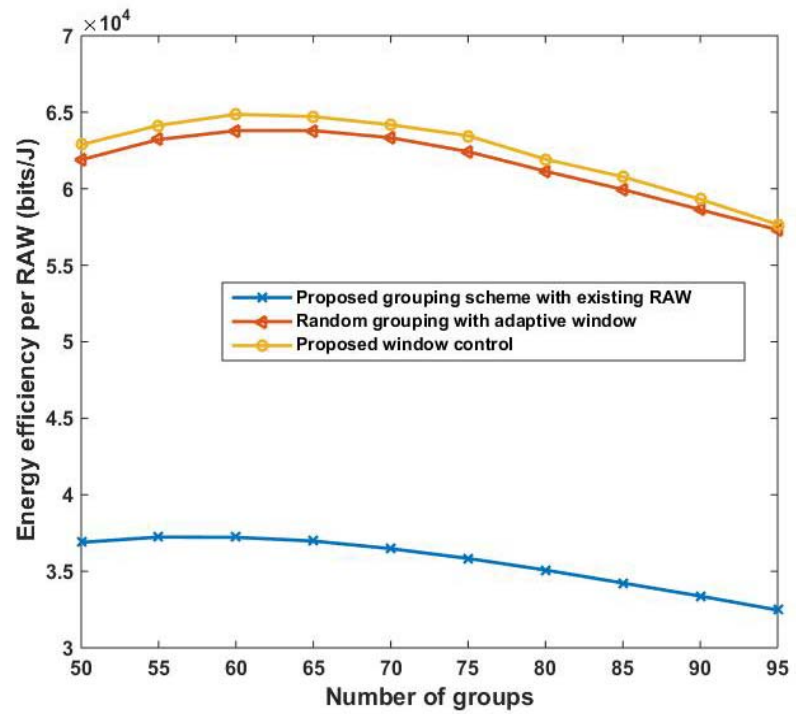

Fig. 5 Uplink energy efficiency per RAW comparison with various number of groups in one scenario $(1,024$ bits/packet).

the collision probability would be high which leads to consume more energy and low data rate, two parameters for low efficiency, thus the trend of curves will go up. However, if the number of groups is too high, the header energy consumption would be high to achieve whole network communications, since scheduling information needs to send multiple times after a short time. So with the increment of the number of groups, the header energy consumption would increase, which is a dominate factor to decrease energy efficiency.

The uplink energy efficiency per RAW varying over diverse number of groups with 1,024 bits/packet is shown in Fig. 5. The proposed window control scheme improves $1.7 \%$ and $77.7 \%$ respectively when comparing with the random grouping with adaptive window and the proposed grouping with the existing RAW. According to Fig. 5, the trend of energy efficiency under three schemes is same with that of 512 bits per packets. Since the data rate increases with the increment of packet size based on the same number of devices, the energy efficiency of 1,024 bits per packet is higher than 512 bits. Though the average energy efficiency of different grouping strategies is similar, the energy efficiency of independent cased could be very poor in the random grouping scheme, which also matches the result of load-balancing grouping in Ref. [12].

Simulation results demonstrate the proposed RAW control scheme could bring about superior fairness and energy efficiency for IEEE 802.11ah uplink communications as efficiently grouping and adaptive window sizes.

\section{Conclusions}

In this paper, a window control scheme is proposed for IEEE 802.11ah networks to optimise uplink communications energy efficiency through clustering and adapting RAW duration (number of slots and internal slot interval) for diverse number of groups and application types. And the retransmission scheme could reuse the empty slots due to random selection of devices. The clustering applied college admissions game to group the devices for super dense networks. The algorithm is built based on Markov Chain and probability theory to evaluate transmission probabilities of various states a device may fall into when sending a packet during one RAW. On account of that, overall energy consumption and data rate are estimated to contribute to energy efficiency. The optimal solution is derived by applying college admissions game and Gradient Descent approach. Simulation results demonstrate that the proposed window control scheme could lead to improvement of energy efficiency and fairness.

\section{References}

[1] Khorov, E., Lyakhov, A., Khorov, A., and Guschin, A. 2014. "A Survey on IEEE 802.11ah: An Enabling Networking Technology for Smart Cities." Computer Communications 58: 53-69.

[2] IEEE Standard for Information Technology Telecommunications and Information Exchange between Systems Local and Metropolitan Area Networks Specific Requirements Part 11: Wireless LAN Medium Access Control (MAC) and Physical Layer (PHY) Specifications Amendment 10: Mesh Networking, 2011.

[3] Wi-Fi Alliance. 2016. "Wi-Fi Alliance Introduces Low Power, Long Range Wi-Fi HaLow.” [Online]. Available: 
https://www.wi-fi.org/news-events/newsroom/wi-fi-allian ce-introduces-low-power-long-range-wi-fi- halow.

[4] Park, C. W., Hwang, D., and Lee, T. 2014. "Enhancement of IEEE 802.11ah MAC for M2M Communications." Communications Letters July: 1151-4.

[5] Vazquez-Gallego, F., Rietti, M., Bas, J., Alonso-Zarate, J., and Alonso, L. 2014. "Performance Evaluation of Frame Slotted-A LOHA with Succesive Interference Cancellation in Machine-to-Machine Networks." European Wireless 2014, 403-8.

[6] Liu, R. P., Sutton, G. J., and Collings, I. B. 2013. "Power Save with Offset Listen Interval for IEEE 802.11ah Smart Grid Communications." 2013 IEEE International Conference on Communications (ICC), 4488-92.

[7] Liu, R. P., Sutton, G. J., and Collings, I. B. 2014. "WLAN Power Save with Offset Listen Interval for Machine to Machine Communications." Wireless Communications 13 (5): 2552-62.

[8] Zhou, Y., Wang, H., Zheng, S., and Lei, Z. Z. 2013. "Advances in IEEE 802.11ah Standardization for Machine-Type Communications in Sub-1GHz WLAN." 2013 IEEE International Conference on Communications Workshops (ICC), 1269-73.

[9] Qutab-ud-din, M., Hazmi, A., Badihi, B., Larmo, A.,
Torsner, J., and Valkama, M. 2015. "Performance Analysis of IoT-enabling IEEE 802.11ah Technology and Its RAW Mechanism with Non-cross Slot Boundary Holding Schemes." 2015 IEEE 16th International Symposium on a World of Wireless, Mobile and Multimedia Networks (WoWMoM), 1-6.

[10] Wang, Y., Li, Y., Chai, K. K., Chen, Y., and Schormans, J. 2015. "Energy-aware Adaptive Restricted Access Window for IEEE 802.11ah Based Networks." 2015 IEEE 26th Annual International Symposium on Personal, Indoor and Mobile Radio Communications (PIMRC), 1253-7.

[11] Specification Framework for TGah, IEEE Std. 802.11-11 1137r15, May 2013.

[12] Chang, T. C., Lin, C. H., Lin, K. C. J., and Chen, W. T. 2015. "Load-Balanced Sensor Grouping for IEEE 802.11 ah Networks." IEEE Global Communications Conference (GLOBECOM), 1-6.

[13] Wang, Y., Chai, K. K., Chen, Y., Schormans, J., and Loo, J. 2017. "Energy-Aware Restricted Access Window Control with Retransmission Scheme for IEEE 802.11ah (Wi-Fi HaLow) Based Networks." IEEE/IFIP WONS 2017, 13th Wireless On-demand Network systems and Services Conference, IEEE/IFIP, 69-76. 